\title{
EL APRENDIZAJE BASADO EN PROBLEMAS (ABP) COMO ESTRATEGIA METODOLÓGICA PARA LA ENSEÑANZA DEL PROCESO ENFERMERERO EN ENFERMERÍA DE PREGRADO
}

\author{
PROBLEM BASED LEARNING (PBL) \\ AS A METHODOLOGICAL STRATEGY FOR TEACHING \\ THE NURSING PROCESS IN UNDERGRADUATE NURSING \\ Ángela Aguayo Puls*, Alejandra Verri Espinosa**, Patricia Rojas Salinas***
}

\section{Resumen}

La aplicación del Proceso Enfermero (PE) requiere del desarrollo de competencias propias del razonamiento o pensamiento crítico. Una de las estrategias pedagógicas que permite la adquisición de esta habilidad es el Aprendizaje Basado en Problemas (ABP) por su vinculación con el aprendizaje significativo. En el caso de esta investigación, el objetivo fue detectar el impacto de la implementación del ABP en la resolución de problemas clínicos, en estudiantes de Enfermería. La muestra estuvo constituida por 44 estudiantes que cursaban la asignatura de Enfermería Médico Quirúrgico. Esta investigación se enmarca en un diseño de modelo mixto y secuencial por etapas. Para ello se analizaron las producciones generadas por los estudiantes, mediante interpretación textual de su discurso escrito, y se

* Magíster en Educación, Gestión y Docencia Universitaria. Facultad de Enfermería. Universidad de Concepción, Chillán, Chile. email: angeaguayo@udec.cl, ORCID https:// orcid.org/0000-0002-4677-3418

** Magíster en Educación, Gestión y Docencia Universitaria, Facultad de Enfermería. Universidad Pedro de Valdivia, Chillán, Chile. email: alejandra.verri@upv.cl, ORCID https://orcid.org/0000-0001-9896-4948

*** Dr. Enseñanza de las Ciencias, Matemática. Cálculo Diferencial e Integral. Académico, Universidad del Bío Bío y Universidad Pedro de Valdivia, Chillán, Chile. email: patricia.rojas.doc@upv.cl, ORCID https://orcid.org/0000-0003-3036-9354 
categorizó el nivel de aprendizaje obtenido a través de la resolución de un caso clínico, ambos análisis fueron sometidos a triangulación complementaria. Los resultados obtenidos demuestran el impacto positivo que logra el $\mathrm{ABP}$ en las habilidades de resolución de problemas clínicos y de transdisciplinariedad, permitiendo la correcta aplicación del Proceso Enfermero. Además, se constató la importancia del trabajo colaborativo como factor de motivación extrínseca, lo que facilitó en los estudiantes la adquisición de conocimientos significativos.

\title{
Palabras clave:
}

Enseñanza, aprendizaje activo, ciencias médicas, competencia profesional, enfermero, estudiante universitario.

\begin{abstract}
The application of the Nursing Process (PE in Spanish) requires the development of critical thinking skills. One of the pedagogical strategies that enables the acquisition of this ability is Problem Based Learning (PBL) due to its link with meaningful learning. The objective of this investigation was to detect the impact of the implementation of PBL in the resolution of clinical problems, in Nursing students. The sample consisted of 44 students taking Medical-Surgical Nursing course. The research is part of a mixed and sequential model design by stages. For this purpose, the products generated by the students were analyzed through textual interpretation of their written discourse, and the level of learning obtained through the resolution of a clinical case was categorized. Both analyzes were subjected to complementary triangulation. The results show the positive impact achieved by PBL in clinical problem-solving skills and transdisciplinarity, allowing the correct application of the Nursing Process. In addition, the importance of collaborative work as an extrinsic motivation factor was verified, which facilitated the acquisition of meaningful knowledge by students.
\end{abstract}

\section{Keywords:}

Teaching, active learning, health sciences, professional competence, nurse, university student. 


\section{Introducción}

La Enfermería se cataloga como una ciencia práctica cuyo objetivo es conocer la realidad de un individuo, familia y/o comunidad, con el fin de poder intervenir y mejorar las condiciones de salud de las personas (Caballero, 2010). En este contexto, se requiere de la aplicación de una metodología ordenada, sistemática y racional, basada en el método científico (González, Blanco, Diz, y López, 2015). Esta metodología se aplica a través de la utilización del Proceso Enfermero (PE) o Proceso de Atención de Enfermería (PAE), el que se define, según Alfaro (1999) como "un método sistematizado de brindar cuidados humanistas centrados en el logro de objetivos (resultados esperados) de forma eficiente" (p. 4).

La aplicación del PAE requiere del desarrollo de competencias propias del razonamiento o pensamiento crítico, para que los profesionales de enfermería puedan emitir juicios clínicos y llevar a cabo acciones basadas en la razón (Caballero, 2010). Por tanto, la docencia actual en Enfermería requiere formar aprendices en el paradigma del pensamiento crítico, fomentando el desarrollo de competencias, como la habilidad de solución de problemas, entendida esta como aquella actividad intelectual que nos permite superar un problema u obstáculo, en cualquier contexto (Roca y Pineda, 2014).

Investigaciones como la de Serdá y Alsina (2016) o la de Suazo, Fuentes, Fernández y Moris (2016) plantean que dentro de las metodologías educativas, orientadas al desarrollo del pensamiento crítico como competencia genérica y el razonamiento clínico como competencia de especialidad, se encuentran el aprendizaje basado en problemas (ABP), el estudio de casos clínicos (ECC), la clase o lección magistral, la lectura guiada, el análisis de textos, la confección de mapas conceptuales, de diarios reflexivos y el portafolio. Todas ellas son empleadas actualmente para potenciar el aprendizaje significativo de los estudiantes de enfermería.

La literatura disponible apunta a que lo más conveniente es utilizar una combinación de estas metodologías. También, se ha demostrado que la utilización de estrategias activas centradas en el aprendizaje autónomo y reflexivo del estudiante favorece a un 
mayor aprendizaje por parte de ellos, respecto de las estrategias tradicionales centradas en la enseñanza y el docente.

En este sentido, Escribano y Del Valle (2008), señalan que el ABP y el ECC son estrategias pedagógicas que promueven el aprendizaje de los estudiantes a través del trabajo autónomo y colaborativo, a la vez que desarrollan competencias transversales como la capacidad de trabajo en equipo y de organización. Además, autores como Roca (2013) y Ley (2014) plantean que el ABP y el ECC se centran en el estudiante como un ente autogenerador de conocimiento, porque favorecen los procesos de reflexión y análisis y facilitan las habilidades metacognitivas de los sujetos. Por consiguiente, la importancia de estudiar una metodología activa como es el Aprendizaje Basado en Problemas será clave para aprender el Proceso Enfermero.

\section{Marco Teórico}

En el siguiente apartado se presentan tres elementos teóricos que permiten sentar las bases de esta investigación. Primero, se dará cuenta de las características generales del Aprendizaje Basado en Problemas (ABP), debido a que es la técnica didáctica y metodológica de enseñanza usada en el desarrollo del estudio. Segundo, se analizará el Proceso de Atención de Enfermería (PAE), para conocer las herramientas que debe aprender el Enfermero con el propósito de lograr su competencia. Tercero, se explicará el Estudio de Caso Clínico para encausar contextualmente el aprendizaje.

\section{Aprendizaje Basado en Problemas}

Barrows (1996) plantea que el Aprendizaje Basado en Problemas (ABP) es un método de aprendizaje y enseñanza, que permite, la adquisición de conocimientos desde el uso de problemas como eje inicial. Éste método está fundamentado en el paradigma constructivista; basado en el aprendizaje por descubrimiento y construcción (Restrepo, 2005). Morales y Landa (2004) por su parte, distinguen 8 
fases en el ABP; las que van desde leer y analizar el problema, realizar una lluvia de ideas, listar lo que se conoce y lo que se desconoce, listar lo que se debe realizar para resolver una situación, definir el problema, obtener la información necesaria y presentar los resultados.

El ABP ha permitido una nueva forma de hacer docencia, cobrando real importancia en la formación de los futuros profesionales de enfermería, principalmente en la enseñanza del PAE, que corresponde a la mirada científica del quehacer de este profesional. Se ha podido constatar que los estudiantes desarrollan habilidades tales como: la comprensión lectora, capacidad de análisis, síntesis y relación de información (Jofré y Contreras, 2013).

El éxito en el uso de ABP requiere que tanto el docente como el estudiante asuman su rol, los que se encuentran claramente definidos. Según Poot (2013), el docente tiene una doble función que incluye la transferencia del conocimiento y un rol de moderador; la actitud se centra en demostrar a sus estudiantes que se enfrentan a un aprendizaje viable y aplicable. Los estudiantes entre tanto deberán integrarse de manera activa, identificando objetivos, estableciendo compromisos, descubriendo, etc., y así se retroalimenta el proceso y se estimula la capacidad de liderazgo, de comunicación y toma de decisiones, la creatividad, el pensamiento crítico y el trabajo en equipo. Cabe señalar que para que esta metodología logre sus objetivos se deben identificar los conocimientos previos sobre el tema; trabajar individualmente y en equipo; presentar posibles soluciones y reflexionar sobre los aprendizajes logrados. Es importante mencionar que el trabajo es recomendado en grupos pequeños, teniendo como punto de partida una situación problemática, que permita analizar y resolver un problema clínico diseñado especialmente para el logro de ciertos objetivos de aprendizaje (González, Carbonero, Lara y Marín 2014).

La responsabilidad de la evaluación en el ABP a diferencia de lo que sucede con el método tradicional, la comparte el tutor con el estudiante, donde este último tiene la responsabilidad de evaluar su aprendizaje y formación, pudiendo autoevaluarse, evaluar a sus compañeros y a su tutor; este proceso evalúa también el trabajo en equipo y los resultados obtenidos de manera cualitativa y formativa. 


\section{Proceso de Atención de Enfermería (PAE)}

Cónsul y Medina (2014) se refieren al Proceso de Atención de Enfermería (PAE) como un método sistemático que conduce a la solución de problemas enfermeros, los cuales se resuelven mediante la aplicación de los cuidados de enfermería, otorgados de manera personalizada a los individuos, familias o comunidades. Al mismo tiempo, el PAE involucra conocimientos teóricos, habilidades procedimentales, consideraciones éticas y toma de decisiones.

Este método contiene fases muy similares a las propuestas por Bransford y Stein (1984) en el método IDEAR para la solución de problemas, cuyas siglas significan identificación del problema, definición y representación del problema, exploración de posibles estrategias, actuación guiada por las estrategias y reflexión o evaluación de los resultados de nuestra actuación (Roca y Pineda, 2014). El método IDEAR, al igual que el PAE, destaca nuevamente la importancia de desarrollar el pensamiento crítico en estudiantes de enfermería y futuros profesionales, para que tomen la elección correcta al momento de dar solución a los problemas detectados. Entendiendo como pensamiento crítico aquel proceso meta cognitivo en el que se elaboran juicios clínicos para resolver problemas a través de la utilización de conocimientos, habilidades y actitudes (Roca, 2013).

Caballero y Arratia (2001), en su investigación para desarrollar el pensamiento crítico en estudiantes de Enfermería, develan la importancia de la búsqueda de información desde todos los puntos de vista, esto permite analizar críticamente situaciones determinadas desde todas las fuentes de información. Además, se hace necesario conocer la forma en que los estudiantes generarán un pensamiento crítico, el que requiere de un aprendizaje activo para la construcción del conocimiento, lo cual involucra observar el valor del concepto adquirido, además de una evaluación constante de su trabajo, complementado con una autoevaluación (Núñez, Ávila y Olivares, 2017). 


\section{Estudio de Caso Clínico}

El método de caso, o estudio de caso clínico, se puede definir como un método activo de aprendizaje centrado en el estudiante, en el cual se parte de una situación problema, que debe ser afrontada por los estudiantes en torno a un aprendizaje concreto, en un tiempo y espacio determinado. El estudio de caso aprueba la integración de la teoría y la práctica, el desarrollo del pensamiento crítico y la mejora de habilidades en la resolución de problemas (Roca, Reguant y Canet, 2015). En este contexto, se le consiente al estudiante de enfermería que aplique el PAE en sus cinco fases, a través del análisis crítico y reflexivo de la situación clínica (real o ficticia) que presenta un individuo, familia y/o comunidad, con la finalidad de plantear las posibles soluciones para los problemas detectados y para los diagnósticos de enfermería enunciados. Por consiguiente, se requiere que el estudiante esté involucrado activamente en su proceso de aprendizaje, tanto en la activación de conocimientos previos como en la adquisición de nuevas nociones que le permitan analizar con fundamentos teóricos la mejor forma de resolver la situación problema.

Autores como Benito y Cruz (2005) y Agudelo (2013), indican que lo más significativo es que este método ofrece la posibilidad de un aprendizaje autónomo, que se complementa con el aprendizaje colectivo que se da en el momento del análisis y discusión del caso en el aula de clase, de tal manera que cobran igual importancia el trabajo individual y el grupal. Por su parte Popil (2011) señala que este método genera en el estudiante un pensamiento crítico frente a las situaciones planteadas, debido a que, al desarrollarlo no está sujeto a respuestas simples o explícitas.

El estudio de caso puede considerarse no solo una estrategia de aprendizaje, sino también una de evaluación, por cuanto el desarrollo de un caso clínico ofrece al docente la posibilidad de apreciar, monitorizar y evaluar cómo sus estudiantes van construyendo su conocimiento, elaborando las relaciones entre datos, aplicando los procesos de análisis y síntesis de información y desarrollando la habilidad de resolución de problemas. Particularmente, en Enfermería se entrega una visión completa de la aplicación del 
PAE, permitiendo determinar el nivel de conocimientos específicos y aplicación del proceso enfermero. Por lo tanto, podemos sostener que el desarrollo de estudio de caso es también una instancia apropiada para evaluar conocimientos particulares o competencias específicas, ya sea de manera formativa o como de resultados (Ojeda, Sangucho, Espinosa, Carrasco y Barbón, 2016).

\section{Metodología}

Este artículo muestra parte de una investigación que se enmarca en el paradigma investigativo mixto (combinando la perspectiva cualitativa y cuantitativa), con un diseño correspondiente al modelo mixto tipo VIII o también denominado "Secuencial por Etapas" (Pereira, 2011). Esto debido a la necesidad de darle profundidad al análisis y abordar el fenómeno desde distintas perspectivas.

El objeto de la investigación es el efecto de estudiar parte de la Unidad de Aprendizaje $\mathrm{N}^{\circ} 5$ del Programa de Asignatura Enfermería Médico Quirúrgico, año 2017, de la carrera de Enfermería de una universidad privada, a través de la estrategia metodológica de Aprendizaje Basado en Problemas (ABP), estrategia que se utilizó por primera vez en la asignatura "Proceso Enfermero, en pacientes con patologías médico-quirúrgicas: Requisito de Autocuidado Universal (RAU) mantenimiento del equilibrio entre la actividad y el descanso".

El universo lo conformaron estudiantes de Enfermería de pregrado cursantes de la asignatura Enfermería Médico Quirúrgico, durante el segundo semestre del año 2017. La muestra está constituida por la totalidad del grupo que cursa como alumno regular la asignatura, en total 44 estudiantes: 38 mujeres y 6 hombres.

El procedimiento de recolección de datos se realizó en tres etapas:

1) Aplicación del Cuestionario de Honey-Alonso de Estilos de Aprendizaje (CHAEA) a la totalidad de los estudiantes, con la finalidad de conformar equipos de trabajo lo más heterogéneos posibles; es decir: se aplicó el test y se analizaron los resultados 
identificando a los estudiantes según el tipo de aprendizaje que mayormente puntúan, y al azar se eligieron los grupos, cautelando que los equipos sean heterogéneos en estilo de aprendizaje (si bien es sabido que todos los individuos pueden desarrollar todo tipo de aprendizajes, se elige la aplicación de este test como estrategia para asegurar heterogeneidad en los grupos de trabajo).

2) Aplicación de la estrategia pedagógica ABP de cuatro fases, durante tres días consecutivos de trabajo colaborativo:

Día 1: etapa de exploración e introducción (cuestionario).

Día 2: etapa de desarrollo y síntesis (confección de cuadros de resumen y análisis de caso clínico).

Día 3: etapa de transferencia (diseño de plan de atención de enfermería).

3) El tercer momento de recolección de datos se llevó a cabo por medio de la aplicación del Estudio de Caso Clínico, el que se realizó una vez finalizada la unidad.

El análisis de resultados, tanto para los productos originados por el ABP (informes, respuestas y notas de los estudiantes) como por el Estudio de Caso Clínico, se llevó a cabo mediante el análisis del discurso escrito en las producciones de los estudiantes (se revisaron todos los insumos confeccionados por los estudiantes, los que eran retirados por el profesor al finalizar cada clase), estos insumos se comparaban con una pauta de corrección creada por el profesor y posteriormente se utilizaba el modelo de triangulación complementario (Creswell, 2008).

La muestra se diseñó aleatoriamente, usando muestreo aleatorio estratificado, para conformar 11 equipos de trabajo heterogéneos, con 4 a 5 integrantes cada uno. A cada equipo de trabajo se le asignó uno de los cuatro $\mathrm{ABP}$ confeccionados, quedando distribuidos de la siguiente manera: $A B P N^{\circ} 1$ (grupos G-1, G-5 y G-9), ABP $N^{\circ} 2$ (grupos G-2, G-6 y G-10), ABP N 3 (grupos G-3, G-7 y G-11) y ABP N 4 (grupos G-4 y G-8). Los once grupos participaron activamente los tres días de actividades planificadas y entregaron la totalidad de los materiales solicitados. 
En este artículo se presenta el análisis de resultados obtenidos al someter a revisión el análisis de proceso y de resultado de los productos entregados por los grupos que trabajaron el ABP $\mathrm{N}^{\circ} 1$ (Ver anexo 1), es decir, se analizan las producciones de G-1, G-5 y G-9.

\section{Resultados}

Al contrastar las respuestas esperadas (pauta creada por el profesor) para el $\mathrm{ABP} \mathrm{N}^{\circ} 1$, con los productos entregados por los estudiantes, se constata que en base a las preguntas de conocimientos previos sobre el sistema osteoarticular y fracturas (preguntas 1 a la 5), los tres grupos alcanzan el requerimiento esperado por sobre el mínimo en las preguntas 3,4 y 5 , demostrando que poseen conocimientos sobre factores de riesgo y consecuencias de las caídas en adultos mayores, como también en la sintomatología de la fractura de cadera. En la pregunta 2 "¿Qué patologías pueden afectar huesos y articulaciones?” dos grupos alcanzan el requerimiento esperado. Destacable es lo que ocurre en la pregunta 1, en que se solicita que mencionen la función de huesos y articulaciones, donde los tres grupos alcanzan solo el requerimiento mínimo.

Al analizar las preguntas 6 a la 11, relativas a conocimientos explorados a través de material entregado durante el desarrollo de la actividad, en lo referente a la coxartrosis de cadera, se puede observar que a pesar de contar con insumos que contienen la información solicitada, los estudiantes no son capaces de lograr los requerimientos esperados, contestando de manera incompleta lo solicitado, la misma situación se observa en las preguntas 6, 7 y 8. En la pregunta 9 que indica: "¿Cuáles son los criterios diagnósticos de Artrosis?", solo el grupo n ${ }^{\circ} 5$ entrega una respuesta completa. En la pregunta 10, respecto de las complicaciones asociadas a la artrosis de cadera, los tres grupos son capaces de identificar complicaciones asociadas a la limitación de la movilidad y el dolor, pero dejan fuera las complicaciones quirúrgicas. Respecto de las alternativas terapéuticas para el tratamiento de la artrosis (pregunta 11), todos los grupos logran el requerimiento esperado. 
Respecto del trabajo, en las etapas de desarrollo o síntesis del $\mathrm{ABP}$, los grupos debían entregar un primer cuadro resumen con las alteraciones y/o manifestaciones clínicas típicas del paciente con artrosis de cadera; un segundo cuadro resumen con las alteraciones y/o manifestaciones clínicas de un caso clínico simulado, relacionado con lo trabajado el día 1, y los diagnósticos de enfermería correspondientes. Los cuadros resumen están configurados según los Requisitos de Autocuidado Universal (RAU), propios del modelo de Dorothea Orem (2001), considerando las manifestaciones presentes en el caso clínico que permiten identificar cuál de estos requisitos se encuentra alterado y los diagnósticos de enfermería según Taxonomía NANDA-I (2013); al analizar el cuadro de los tres grupos es posible observar tanto similitudes como diferencias. Los tres grupos logran identificar la sintomatología clásica de la Artrosis asociada a la alteración de los RAU: Mantenimiento del equilibrio entre la actividad y el descanso, y entre la soledad e interacción social. También logran identificar que no existe alteración del RAU aporte suficiente de líquidos. Las diferencias se pueden observar principalmente en la relación de sintomatología relacionada con el RAU: aporte suficiente de alimentos, en donde dos grupos identifican alteraciones, pero lo hacen desde distintas miradas; el grupo G-1 plantea que puede estar alterado a consecuencia de la patología articular y el grupo G-5 plantea la alteración del RAU por presencia de factores predisponentes, el grupo G-99 no identifica alteraciones asociadas a este RAU.

Se destaca que en los tres grupos existen confusiones respecto de lo relativo a manifestaciones que permiten determinar alteración del RAU, prevención de peligros para la vida, el funcionamiento y el bienestar humano, y los requerimientos relacionados con el RAU, promoción de la normalidad.

Al analizar los cuadros resúmenes entregados por los grupos de trabajo se puede observar que todos poseen respuesta correcta, según pauta, en las manifestaciones asociadas a los RAU: aporte suficiente de aire, de alimentos y mantenimiento del equilibrio entre la actividad y el descanso. En la asociación de las manifestaciones del caso clínico con el resto de los RAU, llama la atención 
que, en el RAU, aporte suficiente de líquidos, los grupos G-1 y G-9 contestan de manera errónea, señalando manifestaciones que no corresponden al RAU. En el RAU, provisión de cuidados asociados con los procesos de eliminación y excreción, ninguno de los grupos identifica la dificultad para desplazarse al baño. Lo mismo ocurre en el RAU, prevención, en donde a los tres grupos les falta identificar uno o más riesgos. Al observar lo ocurrido en el RAU, promoción, ninguno menciona la Hipertensión Arterial en tratamiento y mencionan actividades de enfermería en vez de manifestaciones del usuario.

Respecto de la etapa de síntesis del ABP, representada a través de la solicitud de plantear los diagnósticos de enfermería que correspondan de acuerdo con el caso clínico presentado, se observa que, al comparar los diagnósticos formulados por los grupos, con los diagnósticos de la pauta de corrección, todos los grupos identifican, en término generales, los diagnósticos de enfermería de obesidad, deterioro de la movilidad, ansiedad, riesgo de caídas y riesgo de nivel de glicemia inestable. Respecto de la etiqueta dolor crónico, se observa que dos grupos plantean equivocadamente la etiqueta "dolor agudo" y un grupo no identifica al dolor como diagnóstico de enfermería. En el caso del diagnóstico, riesgo de retraso en la recuperación quirúrgica, relacionado con $(\mathrm{r} / \mathrm{c})$ diabetes mellitus sin tratamiento, ningún grupo identifica el riesgo en el paciente, aun cuando identifican como problema y etiqueta diagnóstica el riesgo de nivel de glicemia inestable. Deterioro de la integridad cutánea, solo el grupo G-1 lo identifica.

En lo particular se puede observar de manera positiva que el grupo G-1 plantea además riesgo de sangrado $\mathrm{r} / \mathrm{c}$ procedimiento quirúrgico, vinculándolo al riesgo intraoperatorio, lo cual es pertinente relacionarlo con el caso clínico; y el grupo G-5 plantea, riesgo de estreñimiento $\mathrm{r} / \mathrm{c}$ disminución de la motilidad gastrointestinal, posiblemente vinculado al proceso post operatorio.

De manera negativa, destaca que los tres grupos tienden a reiterar problemas del usuario con distintas etiquetas diagnósticas, no se observa orden y/o jerarquización en los diagnósticos planteados y aparecen diagnósticos con manifestaciones que no existen en el 
caso clínico, y que posiblemente son planteados pensando en la sintomatología y/o complicaciones asociadas a la patología específica y no a la situación clínica dada.

El tercer día, se trabaja en la etapa de transferencia del ABP, para lo cual se requiere que los grupos elaboren un plan de cuidados enfermeros, aplicando el Proceso Enfermero para un caso clínico simulado (el mismo del día anterior), en base a 4 etiquetas diagnósticas entregadas por el tutor del ABP.

Al analizar la primera etiqueta diagnóstica con la que trabajarán los estudiantes: "deterioro de la movilidad física", podemos apreciar que los tres grupos desarrollan un planteamiento del factor relacionado y de las manifestaciones de manera correcta y pertinente. En el análisis del objetivo se observa que el grupo G-1 lo plantea de manera coherente con el diagnóstico de enfermería, centrado en el usuario, indicando la acción a realizar, el objeto de acción y tiempo. $\mathrm{Al}$ analizar los indicadores se observa que los tres grupos abordan uno o más indicadores pertinentes con el objetivo planteado. Sin embargo, los tres grupos incluyen dentro de los indicadores "disminución o ausencia de dolor", lo cual no se relaciona directamente con la etiqueta diagnóstica ni con el objetivo planteado. Respecto de las intervenciones se puede sostener que todos consideran la administración de analgésicos y la planificación de ejercicios. Solo el grupo G-1 planifica educación al usuario, en relación con la movilidad física. Ninguno de los grupos planifica intervenciones destinadas a medir el logro del objetivo.

Respecto del desarrollo de la etiqueta diagnóstica "ansiedad", los tres grupos plantean factores relacionados y manifestaciones acordes con lo esperado. El planteamiento del objetivo cumple con la coherencia, está centrado en el usuario e indica el objeto de acción. En lo relativo al tiempo en que se debe lograr el objetivo, solo el grupo G-5 es realista, dada la naturaleza del objetivo. Los tres grupos plantean indicadores pertinentes con el objetivo e intervenciones destinadas a la solución del problema, a través de terapias no farmacológicas, y orientadas a tratar la causa de la ansiedad. Ninguno aborda intervenciones destinadas a evaluar el logro del objetivo.

Al comparar la etiqueta "riesgo de úlcera por presión (UPP)" se 
observa que los tres grupos plantean como factor relacionado la "alteración de la movilidad" y todos omiten el factor condicionante de la edad extrema. En el desarrollo del objetivo todos lo hacen correctamente, al igual que al plantear los indicadores. Los grupos G-1 y G-9 además enuncian como indicadores la presencia de factores protectores de piel indemne, como "piel hidratada" y "llene capilar menor de 3 segundos". Las intervenciones planteadas por los tres grupos cumplen los requisitos de permitir la solución del problema, tratar o actuar sobre la causa y evaluar el logro del objetivo propuesto. Destaca que todos planifican frecuencia de actividades que no corresponden con el ideal ni con la realidad, hecho que pudiese explicarse por la falta de experiencia en la ejecución de estas actividades.

En el plan de cuidados diseñado para la etiqueta "riesgo de retraso en la recuperación quirúrgica”, se observa que los tres grupos plantean un factor relacionado en correspondencia con la etiqueta entregada. Los alumnos del grupo G-9 además, relacionan el problema con la obesidad y el deterioro de la movilidad; este mismo grupo logra desarrollar un plan de cuidados coherente y apropiado, ya que plantean objetivos coherentes con el diagnóstico de enfermería, indicadores pertinentes con el objetivo planteado e intervenciones que permiten la solución del problema, actuar sobre la causa y medir los indicadores. Los otros grupos plantean objetivos de manera semi coherente con el diagnóstico e incumplen los criterios establecidos para la formulación de objetivos (planteado en positivo y ser medible). Los indicadores que plantean son pertinentes pero las intervenciones son incompletas; en un grupo (G-1) solo se abordan intervenciones orientadas a medir los indicadores.

\section{Discusión}

Esta investigación coincide con lo planteado por Caballero (2010), quien señala que la aplicación del Proceso de Atención de Enfermería, necesita del desarrollo de competencias que involucren el pensamiento crítico, de manera tal que se entregue una atención 
basada en un método sistemático de cuidados humanistas, centrado en el logro de objetivos. Junto con lo anterior, los resultados de este trabajo coinciden con los planteamientos de Escribano y Del Valle (2008) y con Roca (2013), quienes plantean la importancia de utilizar estrategias pedagógicas que promuevan el trabajo autónomo y colaborativo, ya que ambos fortalecen los procesos de análisis por parte de los estudiantes.

A pesar de lo anterior, Medina y Prado (2009) sostienen que el profesorado no está siempre de acuerdo con formar aulas participativas. Por ello, el presente estudio busca aportar otra perspectiva del proceso de enseñanza y aprendizaje, destacando la importancia de generar vínculos entre profesores y estudiantes, para que ambos acepten la reflexión y la discusión de conceptos, de forma tal que el uso de una estrategia como el Aprendizaje Basado en Problemas sea un aporte para la educación superior.

\section{Conclusiones}

Conformar equipos de estudiantes, intencionando la heterogeneidad de los integrantes, permitió que estos se organizaran de manera eficiente y equitativa, favoreciendo la dinámica de trabajo. En relación con la aplicación del ABP, podemos sostener que fue desarrollado de manera positiva por los estudiantes, permitiendo que se activaran los conocimientos previos mediante el diálogo y el rescate de la información, a partir de los insumos entregados por el tutor. Insumos que, a su vez, fueron apropiados para poder contestar el cuestionario solicitado.

En la etapa de desarrollo y síntesis de conceptos se evidenció que los estudiantes lograron generar sus propias interpretaciones del fenómeno, revelando destrezas importantes en la explicación de sucesos. Se observaron dificultades para identificar las manifestaciones de las alteraciones de los RAU sobre prevención de peligros y promoción de la normalidad en las tablas confeccionadas, y en identificar los problemas del usuario del caso clínico presentado, 
aun cuando lograron aplicar la taxonomía NANDA-I para la elaboración de los diagnósticos.

La etapa de transferencia ha sido evaluada por los investigadores de manera muy positiva, debido a que los estudiantes lograron elaborar diagnósticos enfermeros a partir de una etiqueta diagnóstica dada, aplicando la Taxonomía NANDA-I en su elaboración. Posteriormente son capaces de aplicar el Proceso Enfermero, elaborando un plan de cuidados completo y pertinente.

Asimismo, se puede hacer referencia a que las metodologías activas representan elementos de motivación para el proceso de enseñanza aprendizaje, lo que se puede confirmar con la buena disposición por parte de los estudiantes para realizar todos y cada uno de los trabajos asignados. Esto se complementa con la alta asistencia a las sesiones de clase, a pesar de no ser una actividad obligatoria ni estar asociada a algún tipo de calificación o estímulo alguno, es decir, el trabajo con el Aprendizaje Basado en Problemas, es una técnica didáctica y metodológica de enseñanza muy efectiva en el aprendizaje de la Enfermería. Por lo tanto, es de esperar que otros investigadores puedan trabajar esta metodología en otros cursos de la carrera e ir alternándola con otras técnicas didácticas.

\section{Referencias}

Agudelo, C. (2013). Uso del caso clínico como herramienta pedagógica para el aprendizaje de sintomas generales en homeopatía [Tesis de Magister, Universidad Nacional de Colombia, Bogotá]. Repositorio Institucional Biblioteca Nacional.

Alfaro, R. (1999). Aplicación del Proceso Enfermero. Guía Paso a Paso. Barcelona, España: Springer Science \& Business.

Benito, Á. y Cruz, A. (2005). Nuevas claves para la Docencia Universitaria en el Espacio Europeo de Educación Superior. (Vol. 10). España: Narcea Ediciones.

Barrows, H. (1996). Problem-based learning in medicine and beyond: A brief overview. New Directions for Teaching and Learning, 68, 3-12.

Bransford, D. y Stein, S. (1984). The Ideal Problem Solver: A Guide for Improving Thinking, Learning and Creativity. New York: W.H. Freemam and Company. 
Caballero, É. (2010). Enfermería: Profesión, disciplina y ciencia. En R. Becerra, É. Caballero y C. Huillín (Eds.). Proceso de Enfermería e Informática para la gestión del cuidado (pp. 58-67). Santiago, Chile: Mediterráneo.

Caballero, É. (2010 b). Pensamiento crítico en enfermería. En R. Becerra, É. Caballero y C. Huillín (Eds.), Proceso de Enfermería e Informática para la gestión del cuidado (pp. 73-81). Santiago, Chile: Mediterráneo.

Caballero, E., y Arratia Figueroa, A. (2001). Evaluación e identificación de destrezas del pensamiento crítico en alumnos de primero y segundo año de la carrera de Enfermería-Obstetra. Santiago de Chile: Escuela de Enfermería, Pontificia Universidad Católica.

Cónsul-Giribet, M., y Medina-Moya, J. L. (2014). Strengths and Weaknesses of Problem Based Learning from the Professional Perspective of Registered Nurses. Revista latino-americana de enfermagem, 22, 724-730.

Creswell, J. (2008). Mixed Methods Research: State of the Art. [Presentación de Power Point]. University of Michigan. Recuperado de: sitemaker.umich. edu/creswell.workshop/files/creswell_lecture_slides.ppt

Escribano, A., y Del Valle, A. (2008). El Aprendizaje Basado en Problemas: una propuesta metodológica en Educación Superior. Madrid, España: Narcea Ediciones.

González, C., Carbonero, M., Lara, F. y Martín, P. (2014). Aprendizaje basado en problemas y satisfacción de los estudiantes de Enfermería. Enfermería Global, 13, 97-103.

González, P., Blanco, J. M., Diz, J. y López, M. (2015). Guía de prácticas clínicas: herramienta educativa para la enseñanza de la metodología enfermera. Revista Enfermería Docente, 104, 3-11

Jofré, C. y Contreras, F. (2013). Implementación de la Metodología ABP (Aprendizaje Basado en Problemas) en Estudiantes de Primer Año de la Carrera de Educación Diferencial. Revista Especial Estudios Pedagógicos, 39, 99-113.

Ley, M. (2014). El Aprendizaje Basado en la Resolución de Problemas y su efectividad en el Desarrollo de la Metacognición. Educatio Siglo XXI, 32, 211-230.

Medina, J. y do Prado, M. (2009) El curriculum de enfermería como prototipo de tejido: racionalidad instrumental y tecnológica. Texto y Contexto - Enfermagem, 18, 617-626.

Morales, P. y Landa, V. (2004). Aprendizaje basado en problemas. Theoría, $13,145-157$. 
NANDA Internacional. (2013). Diagnósticos Enfermeros. Definiciones y Clasificación 2012-2014. Barcelona: Elsevier.

Núñez, S., Ávila, J. y Olivares, S. (2017). El desarrollo del pensamiento crítico en estudiantes universitarios por medio del Aprendizaje Basado en Problemas. Revista Iberoamericana de Educación Superior, 23, 84-103.

Ojeda, A., Sangucho, E., Espinosa, C., Carrasco, A. y Barbón, O. (2016). El rol de los casos clínicos publicados en la evaluación del desarrollo de procesos eficaces de razonamiento médico. Revista Cubana de Reumatología, 18, 281-286.

Orem, D. (2001). Nursing: Concepts of Practice. (6th ed.) St. Louis, MO: Mosby.

Pereira, Z. (2011). Los diseños de método mixto en la investigación en educación: Una experiencia concreta. Revista Electrónica Educare, 15, 15- 29.

Poot, C. (2013). Retos del aprendizaje basado en problemas. Revista Enseñanza en Investigación en Psicología, 18, 307-314.

Popil, I. (2011). Promotion of Critical Thinking by Using Case Studies as Teaching Method. Nurse Education Today, 31, 204-207.

Restrepo, B. (2005). Aprendizaje basado en problemas (ABP): Una innovación didactica para la enseñanza universitaria. Revista Educación y Educadores, 8, 9-19.

Roca, J. (2013). El desarrollo del pensamiento crítico a través de diferentes metodologías docentes en el grado en Enfermería. Barcelona: Universidad Autónoma de Barcelona.

Roca, J. y Pineda, P. (2014). Metodologías docentes para el desarrollo de la competencia "solución de problemas" en estudiantes de enfermería. Procedia - Social and Behavioral Sciences, 139, 358-365.

Roca, J., Reguant, M., y Canet, O. (2015). Aprendizaje basado en problemas, estudio de casos y metodología tradicional: una experiencia concreta en el grado en enfermería. Procedia - Social and Behavioral Sciences, 196, 163-170.

Serdá, B. y Alsina, A. (2016). La transmisión de conocimientos versus el aprendizaje autónomo y autorregulado: efectos en el aprendizaje de los estudiantes universitarios. Revista del CIDIU, 3, 1-10.

Suazo, N., Fuentes, O., Fernández, B. y Moris, M. (2016). Aprendizaje Basado en Problemas (ABP) para estudiantes de $3^{\circ}$ año de Enfermería en la Universidad de Talca, 2014. Revista de Educación en Ciencias de la Salud, 13, 37-44. 


\section{Anexos}

$\mathrm{ABP} \mathrm{N}^{\circ} 1$ : Artrosis de Cadera

\section{ABP CASO 1}

\section{Contexto:}

a. Fracturas y caídas en el adulto mayor.

b. Artrosis de cadera.

\section{Día 1:}

\section{Cuestionario}

1. ¿Cuál es la función de huesos y articulaciones?

2. ¿Qué patologías pueden afectar huesos y articulaciones?

3. ¿Qué factores de riesgo favorecen las caídas en el adulto mayor?

4. ¿Qué consecuencias generan las caídas en los adultos mayores?

5. ¿Qué síntomas presentará un paciente con fractura de cadera?

6. ¿Qué es la osteoartrosis (OA)?

7. ¿Cuáles son los factores de riesgo para OA?

8. ¿Cuál es la sintomatología del paciente con artrosis de cadera?

9. ¿Cuáles son los criterios diagnósticos de artrosis?

10. ¿Cuáles son las complicaciones asociadas a la artrosis de cadera?

11. ¿Qué alternativas terapéuticas existen para la artrosis de cadera?

Qo: ¿Qué papel desempeña la osteoporosis en la ocurrencia de artrosis y fractura de cadera y cómo estas influyen en la funcionalidad?

\section{Insumos requeridos}

a. Noticia: "Chile es el tercer país de Latinoamérica con más Artrosis" (La Segunda Online, 14 de febrero de 2013).

b. MINSAL, 2009. Guía Clínica Tratamiento médico en personas de 55 años y más con Artrosis de cadera y/o rodilla, leve o moderada. Flujogramas de manejo médico y de resolución quirúrgica (páginas 4 y 5), 
Confirmación diagnóstica (página 12 - 14).

c. Delgado Morales y otros, 2013. "Osteoporosis, caídas y fractura de cadera. Tres eventos de repercusión en el anciano", Revista Cubana de Reumatología, volumen $X V$, número 1.

d. Rozadilla Sacanell y otros. Artrosis de cadera. Disponible en: http:// www.jano.es/ficheros/sumarios/1/62/1433/31/1v62n1433a13031480pdf001.pdf

Día 2:

\section{Requisitos}

1. Identifique los RAU alterados y la sintomatología correspondiente, en el paciente que presenta Artrosis de cadera. Confeccione cuadro resumen $n^{\circ} 1$.

2. Identifique los RAU alterados y las manifestaciones asociadas, de acuerdo con el caso clínico entregado. Confeccione cuadro resumen $\mathrm{n}^{0} 2$.

3. Elabore los diagnósticos de enfermería para el caso clínico entregado.

\section{Insumos}

a. Caso clínico $n^{\circ} 1$ : paciente con Artrosis de cadera.

b. Texto de apoyo: NANDA-I

\section{Caso clínico $\mathrm{N}^{\circ} 1$}

\section{Antecedentes clínicos}

Usuaria de sexo femenino de 72 años de edad, casada con 3 hijos. Vive sola con su esposo, en el tercer piso de un edificio de departamentos, sin ascensor. Es autovalente y activa. Con antecedentes mórbidos de DM tipo 2, sin tratamiento farmacológico, e HTA en tratamiento con Enalapril.

\section{Evaluación de Enfermería}

Anamnesis:

Paciente refiere que hace 6 meses comenzó con dolor en zona 
inguinal y muslo izquierdo, asociado a esfuerzos moderados. Acudió al médico, quien le diagnosticó Artrosis de cadera izquierda, le indicó tratamiento con AINEs por 5 días y la derivó a rehabilitación kinésica. Posteriormente tuvo remisión parcial e intermitente de las molestias hasta hace 1 mes, en que comienza a presentar claudicación intermitente de la extremidad inferior izquierda.

Actualmente, refiere rigidez articular de cadera izquierda, que desaparece parcial y paulatinamente a medida que inicia sus actividades diarias. Refiere sentirse muy ansiosa por la posible intervención quirúrgica que le informaron debiese realizarse en los próximos días.

Signos vitales:

$\mathrm{P} / \mathrm{A}: 132 / 84 \mathrm{mmHg}$

FC: 62 lat/min

FR: 18 lat $/ \mathrm{min}$

Sat O2: $97 \%$

$\mathrm{T}$ ax: $36.2^{\circ} \mathrm{C}$

Exámenes sanguíneos:

Sodio: $143 \mathrm{mEq} / \mathrm{L}$

Potasio: $4.9 \mathrm{mEq} / \mathrm{L}$

Glicemia: $112 \mathrm{mg} / \mathrm{dl}$

HbA1c: $7.5 \%$

PCR: $0.5 \mathrm{mg} / \mathrm{L}$

Examen físico:

Paciente en cama, orientada en tiempo y espacio, atenta al entorno. Piel y mucosas hidratadas, buena higiene. IMC 30.5 $\mathrm{kg} / \mathrm{m} 2$. No se observan signos congestivos ni de dificultad respiratoria. Abdomen blando, depresible e indoloro. Extremidades superiores bien perfundidas, movilidad conservada. Extremidad inferior izquierda con escasa movilidad, presentando intenso dolor (7/10) al mínimo movimiento, el cual se irradia a la zona lumbar y rodilla izquierda. Ambas extremidades inferiores se observan bien perfundidas, con pulsos presentes. Dorso con ligero enrojecimiento a nivel sacro. Diuresis positiva de aspecto normal. Deposiciones normales hace un día. 
Hipótesis médica

Coxartrosis de cadera izquierda

Indicaciones médicas:

Reposo relativo.

Régimen común.

Ketoprofeno $100 \mathrm{mg} / 8 \mathrm{hrs}$ iv.

Tramitar orden de pabellón.

Exámenes preoperatorios.

Día 3:

\section{Requisitos}

Diseñe un plan de cuidados enfermero para las etiquetas diagnósticas que le serán entregadas. Este plan de cuidados debe contener:

a. Etiqueta diagnóstica entregada.

b. Factor relacionado.

c. Manifestaciones.

d. Objetivo.

e. Indicadores.

f. Intervenciones.

\section{Insumos}

Caso clínico n ${ }^{\circ} 1$ : paciente con Artrosis de cadera. Etiquetas diagnósticas seleccionadas (4).

- Deterioro de la movilidad física

- Ansiedad

- Riesgo de UPP

- Riesgo de retraso de la recuperación quirúrgica 